\title{
AFECÇÕES LARÍNGEAS, TEMPOS MÁXIMOS DE FONAÇÃO E CAPACIDADE VITAL EM MULHERES COM DISFONIA ORGANOFUNCIONAL
}

\section{Laryngeal disorders, maximum phonation times and vital capacity in women with organofunctional dysphonia}

\author{
Carla Aparecida Cielo (1), Bruna Franciele da Trindade Gonçalves (2), \\ Joziane Padilha de Moraes Lima ${ }^{(2)}$, Mara Keli Christmann ${ }^{(3)}$
}

\begin{abstract}
RESUMO
Objetivo: verificar e correlacionar os tempos máximos de fonação (TMF) de vogais, a capacidade vital (CV) e os tipos de afecções laríngeas (AL) de mulheres com disfonia organofuncional (DOF). Método: pesquisa retrospectiva, transversal, exploratória, não experimental, quantitativa, com banco de dados de medidas de TMF [a, i, u], de CV e de AL de mulheres com DOF; e os testes estatísticos Qui- quadrado e exato de Fisher, para verificar as diferenças entre as variáveis e suas relações e o teste binomial, a fim de verificar a significância de proporção ou percentual da análise descritiva, com $p<0,05$. Resultados: a maioria $(22 ; 75,86 \%)$ apresentou TMF significantemente reduzidos $(p=0,0053)$ e sete $(24,14 \%)$ TMF normais. A CV normal foi estatisticamente significante $(p=0,0001)$ (26; $89,66 \%)$, mas três mulheres $(10,34 \%)$ a apresentaram reduzida. Houve predomínio significante dos nódulos vocais ( $p=0,0016)(22 ; 75,86 \%)$, seguido de edema de Reinke $(6 ; 20,69 \%)$ e de pólipo vocal $(1 ; 3,45 \%)$. Dentre as 22 mulheres $(75,86 \%)$ que mostraram TMF reduzidos, houve predomínio com CV normal (19; 86,36\%), embora sem significância estatística $(p=0,558)$. Das mulheres com TMF normais, todas apresentaram CV normal $(7 ; 100 \%)$. A maioria com DOF apresentou CV normal, embora sem significância estatística $(p=0,199)$. Conclusão: nas mulheres com DOF deste estudo, os TMF reduzidos, a CV normal e a presença de nódulos vocais foram significantes e não houve relação entre as variáveis TMF, CV e AL.
\end{abstract}

DESCRITORES: Voz; Fonação; Pregas Vocais; Distúrbios da Voz

\section{INTRODUÇÃO}

A voz é o principal meio pelo qual a comunicação interpessoal é estabelecida, desta forma,

(1) Fonoaudióloga; Professor Adjunto do Curso de Graduação em Fonoaudiologia e do Programa de Pós-Graduação em Distúrbios da Comunicação Humana, Universidade Federal de Santa Maria - UFSM, Santa Maria, Rio Grande do Sul, Brasil; Doutorado em Linguística Aplicada pela Pontifícia Universidade Católica do Rio Grande do Sul.

(2) Acadêmica do Curso de Graduação em Fonoaudiologia da Universidade Federal de Santa Maria - UFSM, Santa Maria, Rio Grande do Sul, Brasil.

(3) Fonoaudióloga; Mestranda do Programa de Pós Graduação em Distúrbios da Comunicação Humana da Universidade Federal de Santa Maria - UFSM, Santa Maria, Rio Grande do Sul, Brasil.

Conflito de interesses: inexistente qualquer alteração na qualidade da mesma, relacionada à frequência, intensidade ou qualidade vocal, gera limitação da comunicação que, por sua vez, se reflete negativamente na qualidade de vida relacionada à voz ${ }^{1,2}$.

As queixas referidas pelos indivíduos que apresentam alguma alteração vocal geralmente são de sensações negativas relacionadas à fonação, as chamadas disestesias vocais, tais como dores no pescoço ou na garganta após uso intenso da voz. Existem fatores que podem contribuir para o surgimento de alterações vocais, como distúrbios alérgicos, faríngeos, orais, nasais, otológicos, pulmonares, digestivos, hormonais e/ou neurovegetativos, além de hábitos como tabagismo, etilismo, exposição a mudanças bruscas de temperatura e comportamentos de uso vocal incorreto ${ }^{3,4}$. 
As alterações vocais decorrentes de lesões nas pregas vocais, originadas prioritariamente pelo uso incorreto da voz, são denominadas disfonias organofuncionais (DOF) $)^{4-9}$ O fonotrauma gerado pelo uso vocal incorreto aumenta o risco de irritação da mucosa da prega vocal e propicia a instalação de lesões laríngeas ${ }^{10-13}$. Este tipo de lesão pode comprometer o fechamento glótico, ocasionando maior escape aéreo durante a fonação e também afetando os valores de sustentação dos tempos máximos de fonação (TMF) ${ }^{9,12,14-18}$.

As principais afecções laríngeas ( $A L)$ organofuncionais encontradas nas avaliações otorrinolaringológicas são nódulos, pólipos vocais, edema de Reinke, úlcera de contato, granuloma e leucoplasias $5,7,9,11,12$, sendo os nódulos vocais a patologia mais encontrada no sexo feminino devido à configuração laríngea que favorece o aparecimento de fenda triangular médio-posterior e também à quantidade reduzida de ácido hialurônico e aumentada de fibronectina, quando comparado ao sexo masculino $7,9,14,19$.

Tais afecções podem ocasionar disfonias, sendo que a avaliação de voz tem como objetivo analisar o comportamento vocal e verificar possíveis fatores que possam estar ocasionando ou agravando as alterações vocais e/ou laríngeas. Tal procedimento é imprescindível para que se possa realizar o diagnóstico por meio da análise conjunta de todos os dados da avaliação e, posteriormente, a intervenção terapêutica adequada ${ }^{9,20,21}$.

Um procedimento objetivo, frequentemente usado na prática clínica, é a avaliação dos TMF, que se refere à emissão sustentada de vogais, fricativos ou de fala encadeada, numa expiração completa em frequência e intensidade habituais ${ }^{9,12,16,22,23}$. $O$ TMF fornece medidas fonatórias quantificadas em segundos, e permite a análise da coordenação entre as forças aerodinâmicas, provenientes da corrente pulmonar expiratória, e as forças mioelásticas da laringe, evidenciando também dados sobre a qualidade da $\mathrm{voz}^{5,9,12,17,20,21,23,24}$.

Outra medida objetiva é a da capacidade vital $(\mathrm{CV})$, quantidade máxima de ar que um indivíduo consegue expirar após uma inspiração máxima ${ }^{9,12}$. Essa medida parece se relacionar com os TMF, pois a emissão sustentada depende, além do controle respiratório e da eficiência glótica, da quantidade de ar pulmonar $9,17,18,21,24$.

Ressalta-se a carência de estudos científicos a respeito da relação entre as variáveis abordadas neste trabalho, embora muito utilizadas na clínica fonoaudiológica. Encontra-se, na literatura atual, pequeno número de estudos correlacionando $\mathrm{AL}$ com a redução dos $\mathrm{TMF}^{17,18}$, havendo também poucos estudos que evidenciem a relação entre tais variáveis e a CV $18,24,25$.

A investigação deste tema apresenta relevância científica, contribuindo com o quadro dos poucos estudos atuais, e relevância clínica para os fonoaudiólogos que atuam na área da voz, pois as relações entre as variáveis analisadas poderão subsidiar o profissional na atuação terapêutica adequada a cada nível da produção vocal, bem como serem utilizadas como parâmetro de normalidade para o sexo feminino e a faixa etária em estudo.

Desta forma, a presente pesquisa pretende verificar e correlacionar os TMF de vogais, a CV e as $\mathrm{AL}$ de mulheres com DOF.

\section{MÉTODO}

Trata-se de uma pesquisa retrospectiva, transversal, exploratória, não experimental e quantitativa, com a utilização de banco de dados de pacientes atendidos em clínica-escola de Fonoaudiologia.

Os critérios de inclusão adotados para seleção dos registros dos sujeitos que compõem a amostra foram: a assinatura do Termo de Consentimento Livre e Esclarecido (TCLE) autorizando a utilização das informações contidas nos prontuários, desde que mantido o sigilo dos dados pessoais; possuir diagnóstico otorrinolaringológico de $\mathrm{AL}$ organofuncional; idades entre 18 e 40 anos, pois nessa faixa etária o aparelho fonador ainda não sofreu a influência das alterações hormonais e estruturais do envelhecimento ${ }^{26,27}$, como também não sofre mais as alterações do período da muda vocal ${ }^{17,26,27}$. possuir dados completos das medidas de CV é TMF, ser do sexo feminino pelo predomínio do mesmo nos registros do banco de dados.

Os critérios de exclusão considerados foram: relato ou diagnóstico de alterações neurológicas, endocrinológicas, psiquiátricas, gástricas; diagnóstico otorrinolaringológico de alterações estruturais mínimas da cobertura das pregas vocais ou de afecção laríngea ou disfonia orgânica; registro de alterações auditivas ao exame audiológico de rotina da clínica-escola; registro de tratamento fonoaudiológico e/ou otorrinolaringológico prévios ao início do tratamento na clínica-escola; ser profissional da voz.

O diagnóstico otorrinolaringológico foi utilizado a fim de verificar o tipo de AL e selecionar apenas as mulheres portadoras de DOF. Os dados de anamnese do banco de dados serviram para verificar a aplicação dos demais critérios de inclusão e de exclusão.

Após a aplicação dos critérios de inclusão e de exclusão, obteve-se uma amostra de 29 mulheres de cujos registros foram utilizadas as medidas de 
TMF das vogais [a, i, u] e da CV, sendo que tais dados foram coletados por diferentes avaliadores em sala acusticamente tratada. Os TMF foram obtidos de maneira padronizada, seguindo o protocolo de coleta da clínica-escola, de modo que os sujeitos foram solicitados a permanecer na postura ereta, com braços estendidos ao longo do corpo, apoiados em ambos os pés e a sustentar três vezes a emissão das vogais [a, i, u] separadamente, em apenas uma expiração em frequência e intensidade habituais, sendo as emissões cronometradas em segundos $^{9,12,18,20,23}$. Os valores foram anotados e foi realizada a média das emissões das vogais [a], [i], [u], utilizando-se a medida de maior valor coletada para cada vogal isoladamente ${ }^{7}$.

Como padrão de normalidade para a média dos TMF, utilizou-se o intervalo de $14,04 \mathrm{~s}$ a $26,96 \mathrm{~s}^{5,9,17}$. Valores abaixo deste intervalo foram considerados como indicativos de escape aéreo à fonação e valores superiores como indicativos de aumento de tensão glótica? .

A CV foi obtida na posição ereta com um espirômetro seco da marca Fami-Itá@, sendo solicitada uma inspiração máxima seguida de uma expiração máxima no aparelho, posicionado na altura do sujeito. A medida da CV utilizada nesta pesquisa foi o maior valor obtido dentre as seis coletas realizadas, sendo três com oclusão nasal e três sem oclusão nasal ${ }^{9,20}$. Como referência de normalidade, utilizaram-se valores iguais ou superiores a $2100 \mathrm{ml}$, sendo que valores inferiores foram considerados abaixo da normalidade para mulheres, sugerindo comprometimento respiratório ${ }^{9}$.

O trabalho foi aprovado pelo Comitê de Ética em Pesquisa da Universidade Federal de Santa Maria/RS.

Instituição de origem (23081008439/2007-16; 00870243000-07) e os sujeitos cujos dados foram utilizados assinaram previamente o TCLE.

Após a tabulação dos dados, foram aplicados testes estatísticos para verificar as diferenças entre as variáveis e suas relações. Foram construídas tabelas de frequência das variáveis categóricas (TMF de vogais, CV e AL), com valores de frequência absoluta $(n)$ e percentual $(\%)$ e foi realizado o teste binomial para verificar a significância de proporção ou percentual da análise descritiva, considerando-se $p<0,01$. Para analisar a relação entre TMF de vogais, CV e AL, foram utilizados o teste Qui-Quadrado ou o teste exato de Fisher, com $p<0,05$.

\section{RESULTADOS}

Tabela 1 - Análise descritiva das variáveis TMF, CV e AL

\begin{tabular}{lccc}
\hline & $\begin{array}{c}\text { Frequência } \\
\text { absoluta (n) }\end{array}$ & $\%$ & p-valor \\
\hline TMF normal & 7 & 24,14 & 0,0027 \\
TMF reduzido & 22 & 75,86 & \\
CV normal & 26 & 89,66 & 0,0001 \\
CV reduzida & 3 & 10,34 & \\
Nódulo & 22 & 75,86 & 0,0016 \\
Edema & 6 & 20,69 & \\
Pólipo & 1 & 3,45 & \\
\hline Teste binomial: $p<0,01$ & & & \\
Legenda: & & & \\
TMF= tempo máximo de fonação & & & \\
CV=capacidade vital & &
\end{tabular}


Tabela 2 -Relação entre as variáveis CV, TMF e AL

\begin{tabular}{|c|c|c|c|c|}
\hline & CV normal & CV reduzida & Total (\%) & p-valor \\
\hline TMF normal & $7(100 \%)$ & $0(0 \%)$ & $7(24,13 \%)$ & \\
\hline TMF reduzido & $19(86,36 \%)$ & $3(13,64 \%)$ & $22(75,86 \%)$ & 0,558 \\
\hline Total & $26(89,65 \%)$ & $3(10,34 \%)$ & $29(100 \%)$ & \\
\hline Nódulo & $21(95,45 \%)$ & $1(4,55 \%)$ & $22(75,86 \%)$ & \\
\hline Edema & $4(66,67 \%)$ & $2(33,33 \%)$ & $6(20,68 \%)$ & 0,199 \\
\hline Pólipo & $1(100 \%)$ & $0(0 \%)$ & $1(3,44 \%)$ & \\
\hline Total & $26(89,65 \%)$ & $3(10,34 \%)$ & $29(100 \%)$ & \\
\hline
\end{tabular}

Legenda:

TMF= tempo máximo de fonação

$\mathrm{CV}=$ capacidade vital

$\mathrm{AL}=$ afecções laríngeas

Tabela 3 - Relação entre os tipos de AL e TMF

\begin{tabular}{lcccc}
\hline & TMF normal & TMF reduzido & Total & p-valor \\
\hline Nódulo & $6(27,27 \%)$ & $16(72,73 \%)$ & $22(75,86 \%)$ & \\
Edema & $0(0 \%)$ & $6(100 \%)$ & $6(20,68 \%)$ & 0,086 \\
Pólipo & $1(100 \%)$ & $0(0 \%)$ & $1(3,44 \%)$ & \\
Total & $7(24,13 \%)$ & $22(75,86 \%)$ & $29(100 \%)$ & \\
\hline
\end{tabular}

Teste exato de Fisher: $\mathrm{p}<0,05$

Legenda:

TMF= tempo máximo de fonação

$\mathrm{AL}=$ afecções laríngeas

Tabela 4 - Relação entre as variáveis TMF, CV e tipos de AL

\begin{tabular}{lccccc}
\hline & $\begin{array}{c}\text { TMF e CV } \\
\text { reduzidos }\end{array}$ & $\begin{array}{c}\text { TMF e CV } \\
\text { normais }\end{array}$ & $\begin{array}{c}\text { TMF } \\
\text { reduzido e } \\
\text { CV normal }\end{array}$ & Total & p-valor \\
& $1(4,55 \%)$ & $6(27,27 \%)$ & $15(68,18 \%)$ & $22(75,86 \%)$ & \\
\hline Nódulo & $2(33,33 \%)$ & $0(0 \%)$ & $4(66,67 \%)$ & $6(20,68 \%)$ & 0,058 \\
Edema & $0(0 \%)$ & $1(100 \%)$ & $0(0 \%)$ & $1(3,44 \%)$ & \\
Pólipo & $3(10,34 \%)$ & $7(24,13 \%)$ & $19(65,51 \%)$ & $29(100 \%)$ & \\
Total & & & & & \\
\hline Teste exato de Fisher: $p<0,05$ & & & & \\
\hline Legenda: & & & & \\
TMF= tempo máximo de fonação & & & & \\
CV=capacidade vital & & & & \\
AL= afecções laríngeas & & & & & \\
& & & & & \\
& & & &
\end{tabular}

\section{DISCUSSÃO}

\section{Avaliação de medidas de TMF e CV e suas correlações}

As avaliações necessárias para a prática clínica na área de Voz constituem-se das avaliações otorrinolaringológica, perceptivo-auditiva da voz, sistema estomatognático, corporal, respiração e análise vocal acústica. Salienta-se, ainda, que tais avaliações são complementares umas às outras e contribuem para o adequado diagnóstico fonoaudiológico, para a elaboração do planejamento terapêutico mais efetivo, bem como para o controle da evolução do caso $0^{5,7,9,12,23}$.

Um dos procedimentos utilizados frequentemente na avaliação da voz é a medida dos TMF $^{9,12,17,20,22,23,28}$, teste objetivo que auxilia de maneira importante na constatação da eficiência glótica sem ser um método invasivo $0^{5,9,12,17,20,21,23,24,28}$. Assim, é possível verificar se as medidas 
encontram-se normais ou alteradas, com base em padrões de normalidade para pares do mesmo sexo e idade, encontrados na literatura (referências de normalidade).

No presente estudo, os TMF da maioria das mulheres foram significantemente reduzidos, evidenciando que as AL de borda de pregas vocais, que compõem o quadro de DOF, interferem no aproveitamento do ar durante a fonação, concordando com outros estudos ${ }^{17,18}$ (Tabela 1).

Outra mensuração objetiva também utilizada na prática clínica é a medida da CV, que quantifica a capacidade pulmonar e está relacionada com a estatura, pois indivíduos maiores possuem caixa torácica maior e, como consequência, maior aporte pulmonar. Outros fatores que afetam a CV são a posição do corpo durante a coleta, força da muscu-

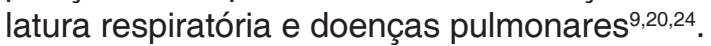

Ressalta-se que a coordenação pneumofônica é um fator importante e pode estar relacionada com os valores da CV pelo fato da provável interferência da última nos TMF, pois os mesmos dependem da quantidade do fluxo aéreo que o indivíduo consegue expirar, além do controle laríngeo. Nesta pesquisa, a CV se encontrou significantemente dentro dos padrões de normalidade, o que sugere boa coordenação pneumofônica por parte das mulheres pesquisadas (Tabela 1).

Além disso, a relação entre CV e TMF faz-se necessária para realizar a distinção entre uma incoordenação do nível fonatório e/ou respiratório ${ }^{5,9,18,24}$. Neste trabalho, essas variáveis não apresentaram relação significante, contudo a maioria percentual das mulheres com DOF e CV normal apresentou TMF reduzidos, concordando com outros estudos em que a CV se apresentou normal, mesmo com os TMF reduzidos em presença de diferentes lesões de borda de pregas vocais, possivelmente em função de os sujeitos desses estudos, bem como deste trabalho, não apresentarem comprometimentos pulmonares ${ }^{17,18}$ (Tabela 4).

Outro fator importante refere-se à escassez de estudos que correlacionem modificações proporcionais entre CV e TMF. No atual estudo, todas as mulheres que apresentaram CV reduzida também apresentaram TMF reduzidos (Tabela 2), concordando com um trabalho realizado com mulheres com diagnóstico de nódulo vocal, no qual se correlacionou tal afecção com TMF e CV, verificando-se que os TMF variavam proporcionalmente à medida da CV encontrada ${ }^{18}$.

Em outra pesquisa, que correlacionou CV e TMF de [e] áfono e de [s] de mulheres normais, verificouse CV e TMF dentro dos padrões de normalidade para o sexo feminino, apontando também para a inter-relação positiva entre essas duas variáveis ${ }^{25}$.
Ainda, com pacientes portadores de asma, quando os TMF estavam reduzidos, a CV também estava e a mesma relação ocorreu com os indivíduos que apresentavam TMF com valores maiores, o que pode ser atribuído ao fato de que os sujeitos avaliados apresentavam comprometimento pulmonar, mostrando que a CV é uma variável que interfere nos valores de TMF ${ }^{24}$.

\section{Relação entre medidas de TMF e CV e AL}

Os TMF podem ser influenciados por diversos fatores, destacando-se a idade, o sexo, características individuais como prática de exercícios físicos ou uso de tabaco, e $\mathrm{AL}^{5,18,24}$. Dentre essas, as lesões de borda de prega vocal interferem diretamente nas medidas dos TMF pelo fato de representarem um impedimento ao adequado fechamento glótico, permitindo maior escape de ar transglótico não sonorizado $5,6,13,15,17,18,29$.

Durante a fonação, é necessário que haja coaptação glótica suficiente para que a mucosa das pregas vocais vibre de maneira homogênea resultando em uma voz agradável. No presente trabalho, como todas as muheres apresentavam DOF, com presença de lesões de borda, os TMF foram reduzidos na maioria significante dos sujeitos (Tabela 3 ), indo ao encontro dos achados da literatura que evidenciam a relação de lesões de borda de pregas vocais com TMF reduzidos $5,6,13,15,17,18$ (Tabela 2 ).

Em pesquisa realizada com 38 mulheres com diagnóstico otorrinolaringológico de nódulos vocais, os TMF foram reduzidos em todas devido à presença da lesão de borda de pregas vocais favorecer o escape aéreo durante a fonação ${ }^{18}$.

Em estudo com 54 indivíduos de ambos os sexos, constatou-se que 46 (85\%) apresentavam diagnóstico médico de nódulos vocais, com predomínio do sexo feminino, sendo que os TMF encontraram-se reduzidos na maior parte dessas mulheres ${ }^{17}$.

Dentre as AL encontradas no presente trabalho, os nódulos vocais apresentaram ocorrência significante (Tabela 1), concordando com outros estudos em que os nódulos em mulheres predominam sobre outras lesões de borda de pregas vocais ${ }^{6,10,15,17,30,31}$.

Os nódulos acometem mais indivíduos adultos do sexo feminino e crianças, devido à configuração laríngea com proporção glótica em torno de 1,0, gerando aumento do ângulo de abertura da comissura anterior das pregas vocais e favorecendo maior impacto entre as mesmas. Tal característica, quando associada ao aumento de tensão muscular, pode gerar fenda triangular médio-posterior que contribui com a formação dos nódulos vocais, pois a hipertensão causa elevação da laringe e fechamento das pregas vocais com vetores de força 
muscular intrínseca desequilibrados, favorecendo maior atrito entre essas estruturas ${ }^{6,7,9}$.

A quantidade reduzida de ácido hialurônico na prega vocal feminina também pode estar relacionada à formação de nódulos, pois a sua concentração reduz 0 atrito durante a vibração por atuar como um amortecedor de impactos na camada mucosa devido a sua propriedade de se ligar a moléculas de água $6,7,9,14,15,17,19$. A quantidade aumentada de fibronectina, glicoproteína de função adesiva encontrada na camada superficial da lâmina própria da mucosa das pregas vocais após abuso vocal, também favorece a formação de nódulos no sexo feminino devido a sua ação cicatrizante e de aderência de fibras ${ }^{7,32}$.

Esses fatores combinados favorecem o aparecimento de nódulos vocais em mulheres e a presença de fenda triangular médio-posterior ou dupla associada aos nódulos. Desta forma, pode ocorrer escape aéreo não sonorizado, levando à diminuição dos TMF, mesmo em presença de CV adequada. O nível fonatório, em presença de nódulos vocais ou outra afecção de borda de pregas vocais, parece ser o principal responsável pela diminuição dos TMF em sujeitos sem comprometimentos respiratórios ${ }^{7,9,12,17,18}$.

\section{CONCLUSÃO}

As mulheres com DOF deste estudo se caracterizaram por apresentar nódulos vocais com TMF reduzidos e CV normal, no entanto, parece não haver relação significante entre as variáveis.

\begin{abstract}
Purpose: to determine and to correlate the maximum phonation times (MPT) of vowels, vital capacity (VC) and laryngeal disorders (LD) for women with benign organic lesions resulting from vocal misuse or abuse (BOL). Method: retrospective, transverse, exploratory, non-experimental, quantitative study, with measurement database of MPT [a, i, u], VC and LD of women with BOL, and Chi-Square statistic and exact tests of Fisher in order to investigate the differences between the variables and their relationships and a binomial test in order to check the significance of proportion or percentage of descriptive analysis, with $\mathrm{p}<0.05$. Results: the majority $(22 ; 75.86 \%)$ showed MPT significantly reduced $(p=0.0053)$ and seven $(24.14 \%)$ normal MPT. The normal VC was statistically significant $(p=0.0001)(26 ; 89.66 \%)$, but three women $(10.34 \%)$ showed it to be reduced. There was significant dominance of vocal nodules ( $p=0.0016)(22 ; 75.86 \%)$, followed by Reinke's edema $(6,20.69 \%)$ and vocal polyp $(1 ; 3.45 \%)$. Among the 22 woman $(75.86 \%)$ which showed reduced MPT, there was a predominance with normal VC $(19 ; 86.36 \%)$, although no statistical significance $(p=0,558)$. All the individuals with normal MPT showed VC normal $(7 ; 100 \%)$. The majority with BOL showed normal VC, although not statistically significant $(p=0,199)$. There was a predominance of vocal nodules and reduced MPT $(16 ; 72.73 \%)$, although not statistically significant $(p=0.086)$. In the correlation of the three variables, most of the subjects presented vocal nodules associated with MPT reduced and normal VC $(15 ; 68.18 \%)$, although not statistically significant. Conclusion: in women with BOL in this study, the reduced MPT, the normal VC and the presence of vocal nodules were significant and there was no relationship between the MPT, VC and LD.
\end{abstract}

KEYWORDS: Voice; Phonation; Vocal Folds; Voice Disorders 


\section{REFERÊNCIAS}

1. Behlau MS, Oliveira G. Recomendação da American Academy of Otolaryngology - Head and Neck Surgery Foundation (AAO-HNSF) sobre "rouquidão" (disfonia). Rev Soc Bras Fonoaudiol. 2009; 14(3): 565-7.

2. Spina AL, Mausell R, Sândalo K, Gusmão R, Crespo A. Correlação da qualidade de vida e voz com atividade profissional. Rev Bras Otorrinolaringol. 2009; 75(2): 275-9.

3. Ferreira LP, Santos JG, Lima MFB. Sintoma vocal e sua provável causa: levantamento de dados de uma população. Rev CEFAC. 2009; 11(1): 110-8.

4. Cielo CA, Finger LS, Roman-Niehues G, Deuschle VP, Siqueira MA. Disfonia organofuncional e queixas de distúrbios alérgicos e/ou digestivos. Rev CEFAC. 2009; 11(3): 431-9.

5. Colton RH, Casper JK. Compreendendo os problemas de voz: uma perspectiva fisiológica ao diagnóstico e ao tratamento. Porto Alegre: Artes Médicas, 1996. p. 1-386.

6. Melo ECM, Brito LL, Osiris Brasil OCO, Behlau M, Danielly Melo DM. Incidência de lesões laríngeas não neoplásicas em pacientes com queixas vocais. Rev Bras Otorrinolaringol. 2001; 67(6): 788-94.

7. Pinho SMR. Avaliação e tratamento da voz. In:__. Fundamentos em fonoaudiologia: tratando os distúrbios de voz. Rio de Janeiro: Guanabara Koogan, 2003; 1-40.

8. Barbosa-Branco A, Romariz MS. Doenças das cordas vocais e sua relação com trabalho. Comun Ciênc Saúde. 2006; 17(1): 37-45.

9. Behlau M. O livro do especialista. Rio de Janeiro: Revinter, 2008. p. 348.

10. Chernobelsky SI. The treatment and results of voice therapy amongst professional classical singers with vocal fold nodules. Logoped Phoniatr Vocol. 2007; 32: 178-84.

11. Behrman A, Rutledge J, Hembree A, Sheridan S. Vocal hygiene education, voice production therapy, and the role of patient adherence: a treatment effectiveness study in women with phonotrauma. J Speech Lang Hear Res. 2008; 51: 350-66.

12. Andrews ML. Avaliação. In _. Manual de tratamento da voz: da pediatria à geriatria. São Paulo: Cengage Learning, 2009. p. 37-110.

13. Barata LF, Madazio G, Behlau M, Brasil O. Análise vocal e laríngea na hipótese diagnóstica de nódulos e cistos. Rev Soc Bras Fonoaudiol. 2010; 15(3): 349-54.

14. Neves BMJ, Neto JG, Pontes P. Diferenciação histopatológica e imunoistoquímica das alterações epiteliais no nódulo vocal em relação aos pólipos e edema de laringe. Rev Bras Otorrinolaringol. 2004; 70(4):439-48.
15. Braga JN, Oliveira DSF, Atherino CCT, Schott TCA, Silva JC. Nódulos vocais: análise anátomofuncional. Rev CEFAC. 2006; 8(2):223-9.

16. Estella M, Robertson J, Radford C, Vagne S, El-Halabi R, Yiu E. Reliability of speaking and maximum voice range measures in screening for dysphonia. J Voice. 2007; 21(4)397-406.

17. Beber BC, Cielo CA, Siqueira MA. Lesões de borda de pregas vocais e tempos máximos de fonação. Rev CEFAC. 2009; 11(1):134-41.

18. Kurtz LO. Tempos máximos de fonação de vogais em mulheres adultas com nódulos vocais. [dissertação]. Santa Maria (RS). Universidade Federal de Santa Maria; 2010.

19. Navas DM, Dias PR. Disfonias funcionais. In: Pinho SR. Fundamentos em fonoaudiologia: tratando os distúrbios da voz. Cap. 5. Rio de Janeiro: Guanabara Koogan; 2003. p. 75-81.

20. Fabron EMG, Santos GR, Omote S, Perdoná GC. Medidas da dinâmica respiratória em crianças de quatro a dez anos. Pró-Fono. 2006; 18 (3): 313-22.

21. Cielo CA, Cappellari VM. Tempo máximo de fonação de crianças pré-escolares. Rev Bras Otorrinolaringol. 2008; 74 (4):552-60.

22. Zhang $\mathrm{Y}$, Jiang JJ. Acoustic analyses of sustained and running voices from patients with laryngeal pathologies. J Voice. 2008; 22 (1): 1-9.

23. Cielo CA, Casarin MT. Sons fricativos surdos. Rev CEFAC. 2008; 10 (3): 352-8.

24. Rossi DC, Munhoz DF, Nogueira CR, Oliveira TCM, Britto ATBO. Relações do pico de fluxo expiratório com o tempo de fonação em pacientes asmáticos. Rev CEFAC. 2006; 8 (4): 509-17.

25. Miglioranzi SL. Capacidade vital e tempos máximos de fonação de /e/ áfono e /s/ em mulheres adultas. [dissertação]. Santa Maria (RS). Universidade Federal de Santa Maria; 2010.

26. Finger LS, Cielo CA. Modificações vocais acústicas produzidas pela fonação reversa. Rev Soc Bras Fonoaudiol. 2009; 14 (1): 15-21.

27. Finger L S, Cielo C A, Schwarz K. Medidas vocais acústicas de mulheres sem queixas de voz $e$ com laringe normal. Braz J Otorhinolaryngol. 2009; 75 (3): 432-40.

28. Speyer R, Bogaardt HCA, Passos VL, Roodenburg NPHD, Zumach A, Heijnen MAM et al. Maximum phonation time: variability and reliability. J Voice. 2010; 24(3):281-4.

29. Gelfer MP, Pazera JF. Maximum duration of sustained /s/ and / $\mathrm{z} /$ and the $\mathrm{s} / \mathrm{z}$ ratio with controlled intensity. J Voice. 2006; 30 (3): 369-79.

30. Nunes, RB; Souza AMV, Duprat AC, Silva MAA, Costa RC, Paulino JG. Análise do trato vocal em pacientes com nódulos, fendas e cisto de prega vocal. Rev Bras Otorrinolaringol. 2009; 188-92. 
31. Bigaton DR, Silvério KCA, Berni KCS, Distefano G, Forti F, Guirro RRJ. Postura crânio-cervical em mulheres disfônicas. Rev Soc Bras Fonoaudiol. 2010; 15 (3): 329-34.
32. Hirschi SD, Gray SD, Thibeault SL. Fibronectin: an interesting vocal fold protein. J Voice. 2002; 16 (3): 310-6.

http://dx.doi.org/10.1590/S1516-18462011005000126

RECEBIDO EM: 28/01/2011

ACEITO EM: 21/03/2011

Endereço para correspondência:

Carla Aparecida Cielo

Rua Pedro Londero, 155 - Dores

Santa Maria - RS

CEP: $97015-121$

E-mail: cieloca@yahoo.com.br 